\title{
Evaluation of cytokine gene expression in psoriasis
}

\author{
Dakshinamurthy Divyapriya ${ }^{1}$, Munisamy Priyadarssini ${ }^{1}$, Sundar Indhumathi ${ }^{1}$, Medha Rajappa ${ }^{1}$, \\ Laxmisha Chandrashekar ${ }^{2}$, Palani Selvam Mohanraj ${ }^{1}$
}

1Department of Biochemistry, Jawaharlal Institute of Post Graduate Medical Education and Research, Puducherry, India ${ }^{2}$ Department of Dermatology, Jawaharlal Institute of Post Graduate Medical Education and Research, Puducherry, India

Adv Dermatol Allergol 2021; XXXVIII (5): 858-865

DOI: https://doi.org/10.5114/ada.2021.110109

\begin{abstract}
Introduction: Psoriasis is a chronic inflammatory disease affecting the skin with an unclear etiological significance. Aim: In this study, we determined the mRNA expression and circulating levels of T helper (Th)/T regulatory (Treg) cytokines in psoriasis and analyzed their association with disease severity and treatment response.

Material and methods: 189 psoriasis patients and 189 controls were recruited. Circulating Th/Treg cytokines (IL-12, IFN- $\gamma$, IL-17, IL-23, TGF- $\beta$ and IL-4) were measured at baseline and at follow-up after 12 weeks of methotrexate treatment by ELISA and their relative mRNA expression at baseline was estimated by quantitative PCR.

Results: We observed increased levels of Th1/Th17 cytokines (IFN- $\gamma$, IL-17, IL-12 and IL-23) and a decrease in levels of Th2/Treg cytokines (IL-4 and TGF- $\beta$ ) in psoriasis patients at baseline, as compared to controls. Further, we observed that there was a significant decrease in Th1/Th17 cytokines, whilst Th2/Treg cytokine levels were significantly increased on follow-up after treatment with systemic metho trexate, as compared to pre-treatment levels. Our results were further confirmed by the significantly higher mRNA expression of Th1/Th17 cytokine genes and significantly lower mRNA expression of Th2/Treg cytokine genes in patients with psoriasis, as compared to controls. A significant positive correlation of Th1/Th17 cytokines was observed with disease severity in cases, whilst Th2/Treg cytokines correlated negatively with disease severity.

Conclusions: Our results show that increased Th1/Th17 cytokines and decreased Th2/Treg cytokines, both at the circulatory and gene expression level, play an important role in the immunopathogenesis and severity of psoriasis.
\end{abstract}

Key words: cytokines, interleukins, gene expression, psoriasis.

\section{Introduction}

Psoriasis is an immune-mediated chronic inflammatory disease of the skin. Its prevalence is $2-3 \%$ of the general population worldwide and may cause significant impairment to the quality of life in the affected person. Though the aetiology of psoriasis remains unclear, several studies have proposed it to be multifactorial with involvement of several genetic and environmental factors leading to its clinical presentation [1]. Though elevated levels of pro-inflammatory cytokines have been reported in the cutaneous lesions and in circulation of psoriasis patients, whether it is the cause or effect of the disease needs to be further explored. Despite advances in the medical field, psoriasis still remains a disease without complete cure [2].

Thelper 1 (Th1) cells act through cell-mediated immune response, especially against bacteria or proto- zoa. Th1 cells are triggered by interleukin-12 (IL-12) to release the effector cytokine, interferon- $\gamma($ IFN- $\gamma)$, which activates the macrophages and induces the expression of class II MHC molecules [3, 4]. An abnormal increase in IFN- $\gamma$ expression has been observed to perpetuate the inflammation in autoimmune diseases such as psoriasis [5]. Th2 cells are mediators of the humoral immune response, especially against helminths. Th2 cells are stimulated by IL-4, which increases the production of effector cytokines like IL-4, IL-10, IL-13, and IL-25 [6]. These activated Th2 cells release IL-4 in a positive feedback mechanism. IL-4 also inhibits Th1/Th17 cells and decrease the cytokine levels of IFN- $\gamma$, by decreasing IL-12 mediated activation of Th1 cells. Previous studies have shown that a defect in IL-4 expression has a pivotal role in the pathogenesis of psoriasis [7].

Address for correspondence: Dr. Medha Rajappa, Department of Biochemistry, Jawaharlal Institute of Postgraduate Medical Education and Research, Puducherry, India, phone: +91-94863-98875, fax: +91-413-2272067, e-mail: linkmedha@gmail.com Received: 9.01.2020, accepted: 31.05.2020. 
IL-17 is another significant inflammatory cytokine secreted by Th17 cells, which are triggered by IL-23 [8]. Immune dysregulation involving imbalance in the Th1/ Th17 related cytokines has been previously shown to have a significant role in various autoimmune diseases including psoriasis, pemphigus and vitiligo $[9,10]$. In psoriasis, IL-17 causes the release of inflammatory cytokines within the epidermal cells, leading to red scaly lesions and chronic inflammation of the skin. Skin biopsy on psoriatic lesions revealed cytotoxic T-cells and IL-17 secreting neutrophils. The IL-23/IL-17 pathway terminates in the excess secretion of pro-inflammatory cytokines, leading to the perpetuation of chronic inflammation in psoriasis [11-13].

Treg cells secrete transforming growth factor- $\beta$ (TGF- $\beta$ ), which inhibits other effector T cells. TGF- $\beta$ inhibits the IL-1 and IL-2 induced proliferation of T cells [14]. FOXP3 is a transcription factor, which is regarded as the master regulator of $T$ cells. It also regulates the activation of T regulatory cells. Mutation in FOXP3 has also been demonstrated in various autoimmune disorders [15].

Although several studies have shown that T-helper and Treg cells have a role in the pathogenesis of psoriasis, there are limited data available regarding the role of various circulatory cytokines and their gene expression in disease progression.

\section{Aim}

In this research, we undertook to determine the circulatory levels of T-helper/Treg cytokines and their specific gene expression to delineate the potential role of the different T-cell subsets in disease progression and further to correlate them with disease severity and treatment response in psoriasis.

\section{Material and methods \\ Setting}

This research was carried out at our hospital, a tertiary care institute in Southern India, as a part of a larger project on psoriasis genetics [16]. The Institute Ethics Committee (Human Studies) approved the study. Written informed consent was collected from all study participants, prior to enrolment in this study. This study conformed to the ethical guidelines in the Declaration of Helsinki and ICMR Ethical Guidelines for Biomedical Research on Human Participants.

\section{Study procedure}

One hundred and eighty-nine patients attending the Psoriasis Clinic in our hospital, aged 18-60 years, diagnosed to have psoriasis and psoriatic arthritis with psoriasis area and severity index (PASI) $>10$ and classified based on international psoriasis consensus classification [17] and CASPAR criteria [18], respectively, were recruited as cases. Patients with inflammatory and infectious diseases, diabetes, pre-existing cardiovascular disease, hypertension, malignancies, smoking and ongoing systemic therapy in the last 3 months were excluded from the study [16]. Age- and gender-matched apparently healthy volunteers who did not have any history of cutaneous disorders, systemic diseases and family history of immunologic disorders were recruited as controls [16].

The sample size was calculated from a similar study in China, which found a difference of $23 \%$ between cases and controls in IFN- $\gamma$ expression [19]. The mean expression among controls was $0.9050 \pm 0.0263$. Hence, we conservatively assumed a margin of $2 \%$ difference in IFN$\gamma$-mRNA expression. In order to detect this difference in the mean value with $90 \%$ power and $5 \%$ significance, the final sample size was calculated as 189 in each group.

\section{Clinical work-up}

The clinical characteristics and detailed family history including the pedigree were collected using a predetermined template. Disease severity was assessed based on PASI scoring [20]. Duration of psoriasis and prior therapies in the past was also recorded. All co-morbidities were recorded. Psoriasis patients were treated with systemic methotrexate at a dose of $7.5 \mathrm{mg} /$ week, increased to $10 \mathrm{mg} /$ week after a month and $15 \mathrm{mg} /$ week after 2 months, depending on the clinical improvement.

\section{Laboratory assays}

$5 \mathrm{ml}$ of blood was collected from the study subjects in EDTA vial for RNA extraction and plasma separation. RNA extraction was done in whole blood by the Trizol method. Plasma was separated and stored at $-80^{\circ} \mathrm{C}$ for measurement of cytokines. The levels of the T helper/Treg cytokines, IL-12, IFN- $\gamma$, IL-17, IL-23, TGF- $\beta$ and IL-4 were assayed using ELISA kits (Diaclone, France) at baseline.

\section{mRNA expression studies at baseline}

RNA was isolated from whole blood using the Trizol method. Purity and RNA concentration was assessed by measuring the absorbance at 260 and $280 \mathrm{~nm}$ using Nanodrop 2000 (Thermo Scientific, United States). 1 mg of RNA was converted into cDNA by using High Capacity cDNA Reverse Transcription Kit (Helini Biomolecules, India). The gene-specific oligonucleotide primers (Eurofins Genomics, India) were used as listed in Supplementary Table S1. CFX96 Real-Time PCR Detection System was used for evaluating the cytokine gene expression levels by using SYBR green and probe master mix (Helini Biomolecules, India). All the experiments were performed in duplicate. Signals were normalized to the housekeeping gene, with $\beta$-actin as the control gene. The relative 
expression of mRNA levels of each gene at baseline was calculated using the CT values of the specific gene and endogenous control by comparative threshold cycle method ( $\Delta \Delta C T$ method) [21].

\section{Follow-up}

Patients were followed up for 3 months to assess the therapeutic response using PASI score, after initiation of systemic methotrexate therapy. $3 \mathrm{ml}$ of blood sample was collected in EDTA vials for estimations of cytokines by ELISA, at the end of 3 months on follow-up.

\section{Statistical analysis}

All categorical variables and discrete variables were presented as frequencies and percentages. The normality of the data was tested using Kolmogorov-Smirnov test. The quantitative data with normal distribution were presented as mean with standard deviation (SD) and median with the interquartile range was used to present the non-Gaussian data. To assess the difference in continuous variables between the groups, appropriate tests were used based on the distribution of data. The relationship of mRNA expression and cytokine levels with disease severity was analysed using Spearman correlation. All statistical analyses were carried out at $5 \%$ leve of significance. A p-value less than 0.05 was considered as significant.

\section{Results}

Supplementary Table S2 depicts the baseline characteristics of the study participants, which have been published previously [16]. We compared the baseline cytokine levels between the psoriasis cases and controls in the study population (Figure 1). We observed that there was a significant elevation in the circulating Th1 and Th17 cytokine levels in psoriasis cases compared to controls, whilst the IL-4 and TGF- $\beta$ levels were significantly decreased in the cases, as compared with control volunteers.

We also correlated the baseline cytokine levels with disease severity and observed that circulating levels of

Table 1. Correlation of cytokine levels with disease severity (PASI)

\begin{tabular}{lcc}
\hline Parameter & $\rho$ value & $P$-value (Spearman correlation) \\
\hline IFN- $\gamma$ & 0.775 & $<0.0001$ \\
\hline IL-17 & 0.745 & $<0.0001$ \\
\hline IL-12 & 0.686 & $<0.0001$ \\
\hline IL-23 & 0.612 & $<0.0001$ \\
\hline IL-4 & -0.772 & $<0.0001$ \\
\hline TGF- $\beta$ & -0.774 & $<0.0001$ \\
\hline
\end{tabular}

pro-inflammatory cytokines, IFN- $\gamma$, IL-17, IL-12 and IL-23 showed a significant positive correlation with PASI, whilst anti-inflammatory cytokines, IL-4 and TGF- $\beta$ correlated negatively with PASI (Table 1).

We studied the cytokine levels in plasma at baseline and at follow-up after 3 months with systemic methotrexate therapy in patients with psoriasis (Figure 2). We observed that the circulating levels of IFN- $\gamma$, IL-17, IL-12 and IL-23 cytokines decreased significantly at 3 months of follow-up, compared to baseline, whilst the circulating levels of IL- 4 and TGF- $\beta$ cytokines increased significantly after treatment.

We also observed that mRNA expression of pro-inflammatory cytokines, IFN- $\gamma$, IL-12, IL-17, and IL-23 were significantly upregulated in psoriasis cases, whilst the anti-inflammatory cytokines, IL-4 and FOXP3 mRNA expression were significantly downregulated in psoriasis cases when compared to controls (Figure 3).

We also correlated the relative mRNA expression with disease severity. Our results revealed that mRNA expression of IFN- $\gamma$, IL-17, IL-12 and IL-23 cytokines correlated positively with PASI $(r=0.653, p<0.0001 ; r=0.722, p<$ $0.0001 ; r=0.585, p<0.0001$ and $r=0.819, p<0.0001)$ and there was a significant negative correlation of IL-4 and FOXP3 mRNA expression with disease severity $(r=$ $-0.818, p<0.0001$ and $r=-0.620, p<0.0001$ respectively) (Table 2).

\section{Discussion}

In this study, we determined the circulating levels of T-helper and T-regulatory cytokines in patients with psoriasis at baseline and after 12 weeks follow-up posttreatment and their specific gene expression at baseline.

In this study, we observed an upregulation in the Th1/ Th17 cytokine levels and downregulation in the Th2/Treg cytokine levels in peripheral blood of psoriasis patients at baseline, as compared to healthy volunteers. We observed that circulating levels of Th1/Th17 cytokines such as IL-12, IFN- $\gamma, \mathrm{IL}-17$, and IL-23 were significantly decreased, whilst the plasma IL-4 and TGF- $\beta$ levels were significantly increased during follow-up after 3 months of systemic methotrexate therapy, compared to baseline

Table 2. Correlation of relative mRNA expression of cytokine genes with disease severity (PASI)

\begin{tabular}{lcc}
\hline Parameter & $\rho$ value & $\begin{array}{c}P \text {-value (Spearman } \\
\text { correlation) }\end{array}$ \\
\hline IFN- $\gamma$ mRNA expression & 0.653 & $<0.0001$ \\
\hline IL-17 mRNA expression & 0.722 & $<0.0001$ \\
\hline IL-12 mRNA expression & 0.585 & $<0.0001$ \\
\hline IL-23 mRNA expression & 0.819 & $<0.0001$ \\
\hline IL-4 mRNA expression & -0.818 & $<0.0001$ \\
\hline FOXP3 mRNA expression & -0.620 & $<0.0001$ \\
\hline
\end{tabular}



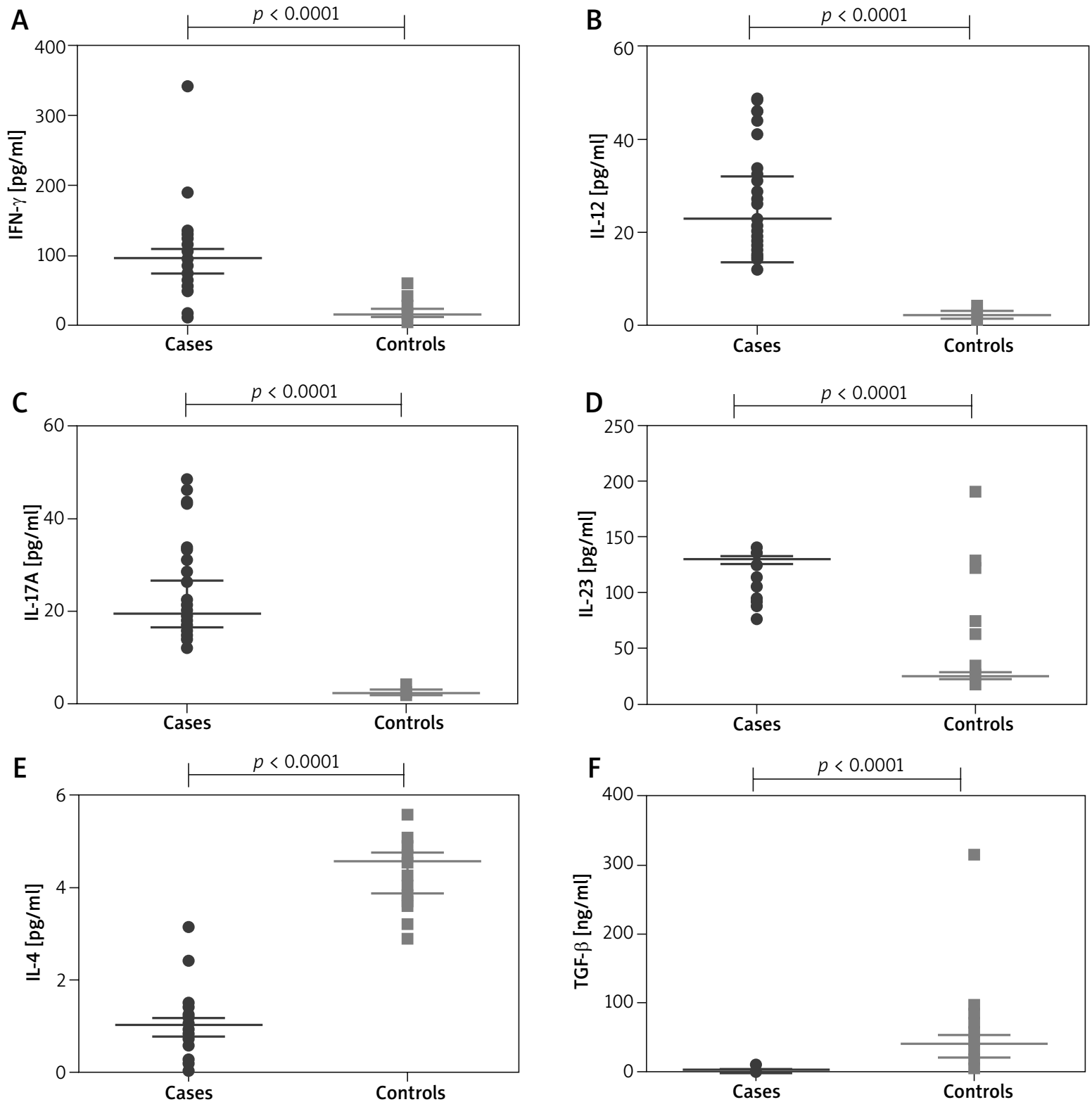

Figure 1. Comparison of cytokine levels in plasma of 189 psoriasis patients and 189 controls. IFN- $\gamma$ (A), IL-12 (B), IL-17 (C), IL-23 (D), IL-4 (E) and TGF- $\beta$ (F). Mann-Whitney test (A-F) was used to analyse these data. Each symbol represents individual samples and horizontal lines show the median values $(\mathbf{A}-\mathbf{F})$

levels. Our results were further confirmed by significantly raised mRNA expression of Th1/Th17 cytokine genes and significantly lowered mRNA expression of Th2/Treg cytokine genes in psoriatic patients as compared to healthy volunteers. We have also observed a significant positive correlation of Th1/Th17 cytokine levels with disease severity, whilst Th2/Treg cytokines correlated negatively with disease severity.

These results suggest that both Th1 and Th17 cytokines have a pro-inflammatory role in the pathogenesis of psoriasis. The decrease in their levels after 3 months of methotrexate monotherapy implies that these cytokines have a direct role in disease progression as well. Similar findings were observed in previous studies, where IFN- $\gamma$ levels have been shown to be increased in patients with psoriasis [5, 22].

Previously it was thought that Th1 cytokines like IFN- $\gamma$ and IL-12 were responsible for the disease progression in psoriasis. However, recent studies show that IL-17 plays a major role in the pathogenesis of psoriasis [11, 23]. Increase in IL-23, resulting in activation of Th17 cells, has been reported as a major event in disease pathogenesis 

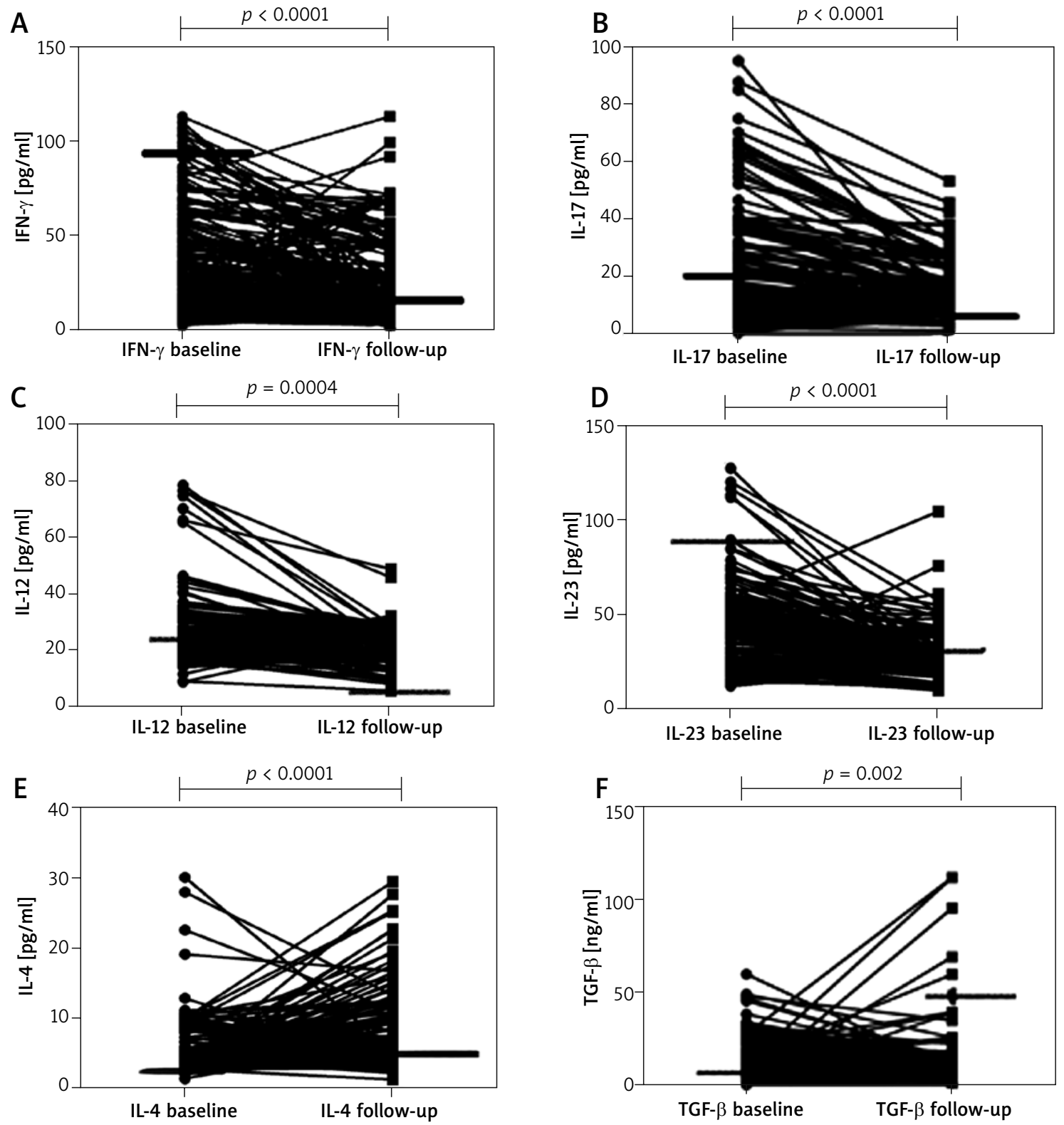

Figure 2. Comparison of pre- and post-treatment cytokine levels in plasma of 189 psoriasis patients and 189 controls. IFN- $\gamma(\mathbf{A})$, IL-17 (B), IL-12 (C), IL-23 (D), IL-4 (E) and TGF- $\beta$ (F). Wilcoxon's signed-rank test (A -F) was used to analyse these data. Each symbol represents individual samples and horizontal lines show the median values (A-F)

$[24,25]$. Studies have shown that both Th1 and Th17 are involved in different phases such as initiation and activation of psoriasis [26, 27].

We observed that IL-4 and TGF- $\beta$, the Th2/Treg related cytokines were significantly lower in cases as compared to controls and the levels showed a significant increase during follow-up after 3 months. This suggests that Th2/Treg cytokines have a regulatory role in the disease progression and increase in Th2/Treg cytokines have a protective effect against the progression of psoriasis.
Prens et al. have shown that there is an increase in IL-4 receptors on epithelial cells of psoriasis, which is in contradiction to our results, but this can be explained by a shift in immune response from Th1 to Th2 as a compensatory mechanism to counteract the increase in IL-1 [28]. Karamehic et al. reported that Treg cells in peripheral blood are higher in psoriasis patients as compared to healthy subjects [29]. Quaglino et al. have also shown that Th1 and Th17 cells were upregulated and Treg cells were downregulated in psoriasis and there was a reversal 
A

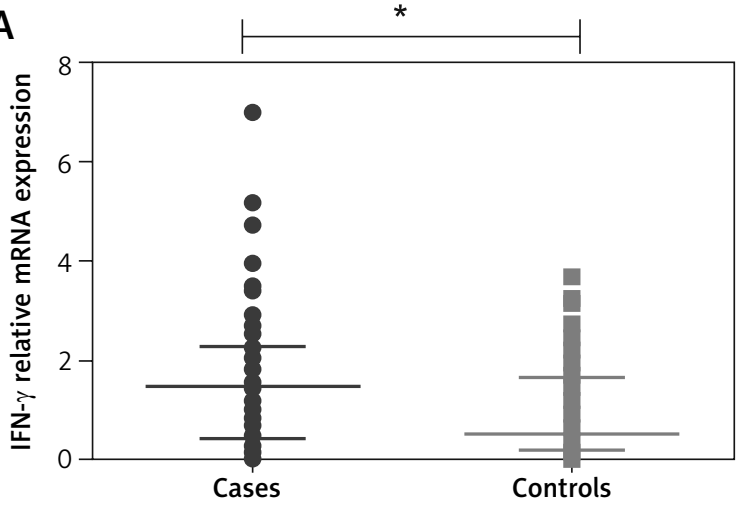

\section{C}

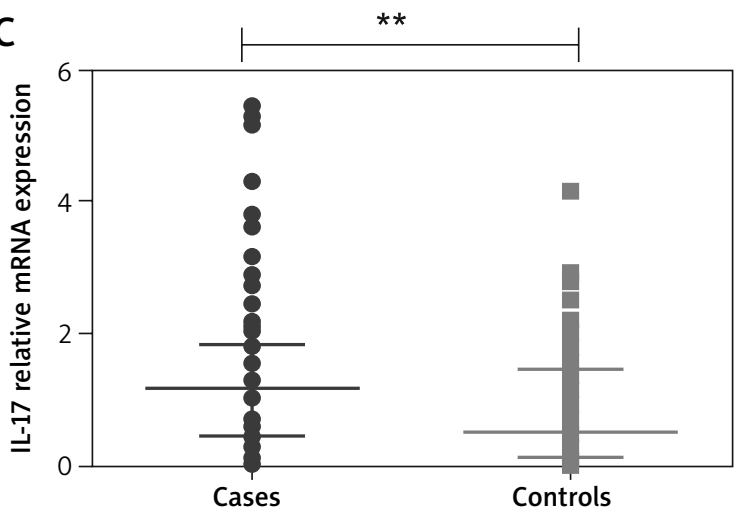

E

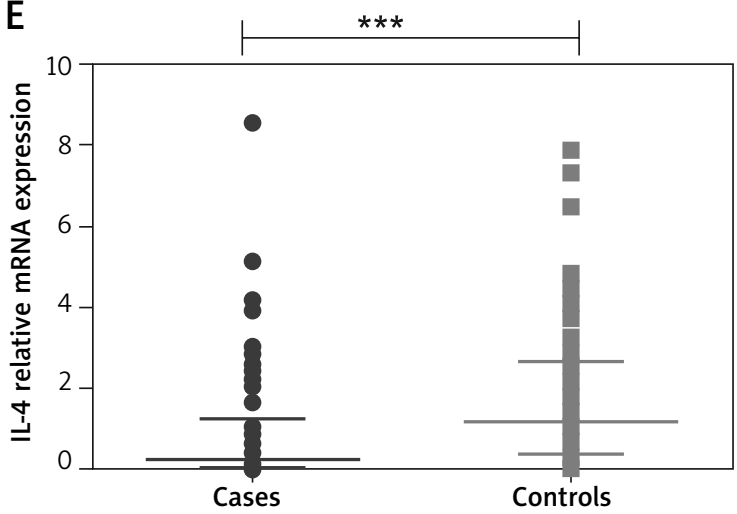

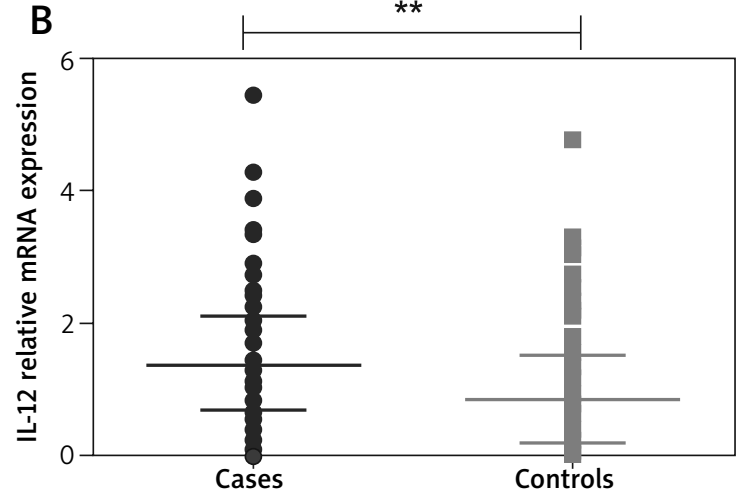
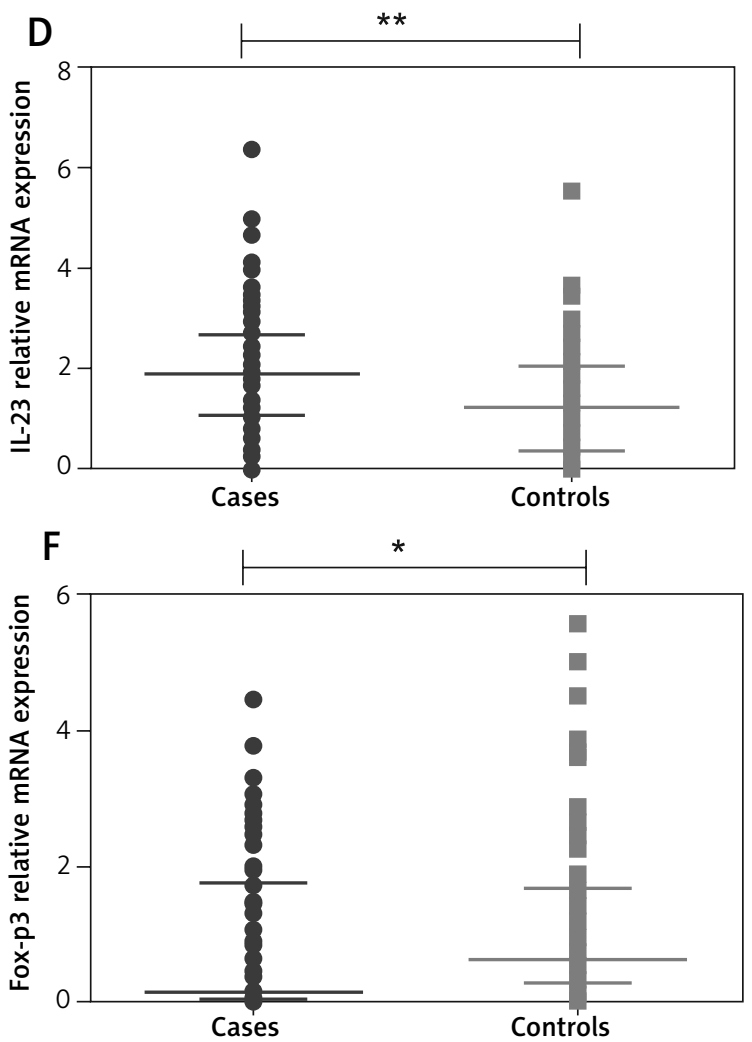

Figure 3. Comparison of relative cytokine gene expression in peripheral blood of 189 psoriasis patients and 189 controls. IFN- $\gamma$ (A), IL-12 (B), IL-17 (C), IL-23 (D), IL-4 (E), FOXP3 (F). Mann-Whitney test was used to analyze these data. Each symbol represents individual samples and horizontal lines show the median values $\left({ }^{*} p<0.05,{ }^{* *} p<0.01,{ }^{* *} p<0.001\right)$

in the levels after treatment [30]. The above results are concordant with the results obtained in our study.

We have also estimated the relative mRNA expression of Th/Treg cytokines in peripheral blood at baseline. Relative mRNA expression studies showed significant upregulation of cytokines IL-12, IFN- $\gamma$, IL-17 and IL-23 and significant downregulation of IL-4, which supports our observed pattern of circulating cytokine levels. The mRNA expression of FOXP3, which is the master regulator of
T-cells, was significantly lower in psoriasis suggesting a decrease in Treg cell related cytokines such as TGF- $\beta$. Recent reports suggest that phenotypic expression of psoriasis is mainly based on the cytokine profile in circulation and it determines the disease severity as well [31-33].

We also correlated the mRNA expression and cytokine levels at baseline with the disease severity. Correlation studies show that there is a positive correlation 
of IL-12, IFN- $\gamma$, IL-17 and IL-23 mRNA expression levels, whilst there is a significant negative correlation of IL-4 and TGF- $\beta$ mRNA expression levels with disease severity assessed by PASI score. Our results are in agreement with the previous study, which also showed that pro-inflammatory cytokines positively correlated with disease severity [5].

Our study is not without limitations. We have not evaluated the pattern of other Th22/Th9 cytokines, which could also contribute to disease pathogenesis. Genome-wide association studies of various cytokine genes in psoriasis in ethnic populations worldwide would facilitate a comprehensive understanding of its genetic basis.

\section{Conclusions}

There is an immunologic imbalance of T cells in psoriasis, associated with upregulation in Th1/Th17 pro-inflammatory cytokines and downregulation of Th2/Treg cytokines both in circulation, as well as at the level of gene expression of their respective cytokines, especially with increasing disease severity. This immune dysregulation, which plays a key role in the disease progression, could probably be reversed by treatment with systemic methotrexate in patients with psoriasis.

\section{Acknowledgments}

Funding by the Department of Biotechnology (DBT), New Delhi, India (Grant number BT/PR6033/ GBD/27/373/2012) in the name of Dr. Medha Rajappa is gratefully acknowledged.

DPD and MP have contributed equally to the study and hence are co-first authors for this research work.

\section{Conflict of interest}

The authors declare no conflict of interest.

\section{References}

1. Hwang ST, Nijsten T, Elder JT. Recent highlights in psoriasis research. J Investig Dermatol 2017; 137: 550-6.

2. Nishibu A, Han GW, Iwatsuki K, et al. Overexpression of monocyte-derived cytokines in active psoriasis: a relation to coexistent arthropathy. J Dermatol Sci 1999; 21: 63-70.

3. Schlaak JF, Buslau M, Jochum W, et al. T cells involved in psoriasis vulgaris belong to the Th1 subset. I Investig Dermatol 1994; 102: 145-9.

4. Abul H, Mahmoud F, Al-Saleh Q, et al. Profiles of activated T lymphocytes in peripheral blood of Kuwaiti psoriasis vulgaris patients. I Dermatol 2002; 29: 202-8.

5. Szegedi A, Aleksza M, Gonda A, et al. Elevated rate of Thelper1 (Th1) lymphocytes and serum IFN- $\gamma$ levels in psoriatic patients. Immunol Lett 2003; 86: 277-80.

6. Guenova E, Skabytska Y, Hoetzenecker W, et al. IL-4 abrogates Th17 cell-mediated inflammation by selective silencing of IL-23 in antigen-presenting cells. Proc Natl Acad Sci USA 2015; 112: 2163-8.
7. Steinke JW, Borish L. Th2 cytokines and asthma - interleukin-4: its role in the pathogenesis of asthma, and targeting it for asthma treatment with interleukin-4 receptor antagonists. Respir Res 2001; 2: 66-70.

8. Miossec P, Korn T, Kuchroo VK. Interleukin-17 and type 17 helper T cells. N Engl J Med 2009; 361: 888-98.

9. Hu Y, Shen F, Crellin NK, Ouyang W. The IL-17 pathway as a major therapeutic target in autoimmune diseases. Ann N Y Acad Sci 2011; 1217: 60-76.

10. Das D, Shamima A, Santosh K, et al. Emerging role of immune cell network in autoimmune skin disorders: an update on pemphigus, vitiligo and psoriasis. Cytokine Growth Factor Rev 2019; 45: 35-44.

11. Martin DA, Towne JE, Kricorian G, et al. The emerging role of IL-17 in the pathogenesis of psoriasis: preclinical and clinical findings. J Investig Dermatol 2013; 133: 17-26.

12. Lowes MA, Suárez-Farińas M, Krueger JG. Immunology of psoriasis. Annu Rev Immunol 2014; 32: 227-55.

13. Mudigonda P, Mudigonda T, Feneran AN, et al. Interleukin-23 and interleukin-17: importance in pathogenesis and therapy of psoriasis. Dermatol Online J 2012; 18: 1.

14. Tiemessen MM, Kunzmann S, Schmidt-Weber CB, et al. Transforming growth factor $\beta$ inhibits human antigen-specific CD4+ T cell proliferation without modulating the cytokine response. Int Immunol 2003; 15: 1495-504.

15. Hori S, Nomura T, Sakaguchi S. Control of regulatory T cell development by the transcription factor Foxp3. Science 2003; 299: 1057-61.

16. Priyadarssini M, Divya Priya D, Indhumathi S, et al. Immunophenotyping of T cells in the peripheral circulation in psoriasis. Br J Biomed Sci 2016; 73: 174-9.

17. Griffiths CE, Christophers E, Barker JN, et al. A classification of psoriasis vulgaris according to phenotype. Br I Dermatol 2007; 156: 258-62.

18. Taylor W, Gladman D, Helliwell P, et al. Classification criteria for psoriatic arthritis: development of new criteria from a large international study. Arthritis Rheumatol 2006; 54: 2665-73.

19. Jiawen L, Dongsheng L, Zhijian T. The expression of interleukin-17, interferon-gamma, and macrophage inflammatory protein-3 alpha mRNA in patients with psoriasis vulgaris. J Huazhong Univ Sci Technolog Med Sci 2004; 24: 294-6.

20. Langley RG, Ellis CN. Evaluating psoriasis with psoriasis area and severity index, psoriasis global assessment, and lattice system physician's global assessment. J Am Acad Dermatol 2004; 51: 563-9.

21. Livak KJ, Schmittgen TD. Analysis of relative gene expression data using real-time quantitative PCR and the 2(-Delta Delta C (T)) Method. Methods 2001; 25: 402-8.

22. Kagami S, Rizzo HL, Lee JJ, et al. Circulating Th17, Th22, and Th1 cells are increased in psoriasis. J Invest Dermatol 2010; 130: 1373-83.

23. Benham H, Norris P, Goodall J, et al. Th17 and Th22 cells in psoriatic arthritis and psoriasis. Arthritis Res Ther 2013; 15: R136.

24. Girolomoni G, Strohal R, Puig L, et al. The role of IL-23 and the IL-23/Th17 immune axis in the pathogenesis and treatment of psoriasis. J Eur Acad Dermatol Venereol 2017; 31: 1616-26.

25. Ogawa E, Sato Y, Minagawa A, Okuyama R. Pathogenesis of psoriasis and development of treatment. J Dermatol 2018; 45: 264-72. 
26. Diani M, Altomare G, Reali E. T helper cell subsets in clinical manifestations of psoriasis. J Immunol Res 2016; 2016: 7692024.

27. Mehta NN, Dagur PK, Rose SM, et al. IL-17A production in human psoriatic blood and lesions by CD146+ T cells. I Invest Dermatol 2015; 135: 311-4.

28. Prens E, Hegmans J, Lien RC, et al. Increased expression of interleukin-4 receptors on psoriatic epidermal cells. Am J Pathol 1996; 148: 1493-502.

29. Karamehic J, Zecevic L, Resic H, et al. Immunophenotype lymphocyte of peripheral blood in patients with psoriasis. Med Arch 2014; 68: 236-8.

30. Quaglino P, Bergallo M, Ponti R, et al. Th1, Th2, Th17 and regulatory $T$ cell pattern in psoriatic patients: modulation of cytokines and gene targets induced by etanercept treatment and correlation with clinical response. Dermatology 2011; 223: 57-67.

31. Chun JH, Yoon YH, Choe YB, et al. Gene expression analysis of inflammatory cytokines in Korean psoriatic patients. Ann Dermatol 2017; 29: 422-6.

32. Zhang HY, Yan KX, Huang Q, et al. Target tissue ectoenzyme CD39/CD73-expressing Foxp3+ regulatory T cells in patients with psoriasis. Clin Exp Dermatol 2015; 40: 182-91.

33. Keijsers RR, van der Velden HM, van Erp PE, et al. Balance of Treg vs. T-helper cells in the transition from symptomless to lesional psoriatic skin. Br J Dermatol 2013; 168: 1294-302. 